\title{
Self-sustained Marangoni Flows Driven by Chemical Reactions
}

\author{
Anne-Déborah C. Nguindjel and Peter A. Korevaar*[a] \\ [a] A.-D. C. Nguindjel, Dr. P. A. Korevaar \\ Institute for Molecules and Materials \\ Radboud University \\ Heyendaalseweg 135, 6525 AJ Nijmegen, the Netherlands \\ E-mail: p.korevaar@science.ru.nl \\ Supporting information for this article is given via a link at the end of the document
}

\begin{abstract}
Out-of-equilibrium chemical systems, comprising reaction networks and molecular self-assembly pathways, rely on the delivery of reagents. Rather than via external flow, diffusion or convection, we aim at self-sustained reagent delivery. Therefore, we explore how the coupling of Marangoni flow with chemical reactions can generate selfsustained flows, driven by said chemical reactions, and - in turn sustained by the delivery of reagents for this reaction. We combine a photoacid generator with a $\mathrm{pH}$-responsive surfactant, such that local UV exposure decreases the $\mathrm{pH}$, increases the surface tension and triggers the emergence of a Marangoni flow. We study the impact of reagent concentrations and identify threshold conditions at which flow can emerge. Surprisingly, we unraveled an antagonistic influence of the reagents on key features of the flow such as interfacial velocity and duration, and rationalize these findings via a kinetic model. Our study displays the potential of reaction-driven flow to establish autonomous control in fuel delivery of out-of-equilibrium systems.
\end{abstract}

\section{Introduction}

Living organisms display a wide range of dynamic functionalities that emerge from chemical reaction networks ${ }^{1}$, molecular assembly pathways ${ }^{2}$ and reaction-diffusion systems ${ }^{3}$. As a hallmark of life, these processes occur under out-of-equilibrium conditions, and are maintained by dissipation of energy via chemical reactions. Over the past decade, many synthetic systems have been built to emulate these out-of-equilibrium dynamics ${ }^{4,5}$ in matter with life-like properties, exemplified by reaction networks that display circadian oscillations $\mathrm{s}^{6-8}$, adaptive responses $^{9}$, self-assembled structures with transient properties mediated by chemical reactions ${ }^{10-18}$, or patterns ${ }^{19-23}$ that selforganize from homogeneous start conditions. These phenomena are typically established in solution and rely on the delivery of chemical reagents by external flow, diffusion ${ }^{24}$ or convection ${ }^{25}$. In biological systems, however, the localized delivery of reagents as a mean to coordinate self-organization of structures, or to control the positioning of a reaction site - is regulated via autonomously operating processes, driven by intricate regulation and feedback mechanisms. In a synthetic context, concentration gradients - which can be regulated via chemical reactions - can provide a driving force for such transfer of reagents. In this regard, mass transfer that is induced by the Marangoni effect ${ }^{26}$ appears as an interesting phenomenon to establish spatial control in outof-equilibrium systems. Indeed, this interfacial flow, driven by surface tension gradients that arise from spatial differences in temperature or concentration of surface active compounds, presents the opportunity to regulate motion at interfaces through chemistry.
Interfacial flows induced by the Marangoni effect have been extensively used to displace objects or fluids ${ }^{27}$. In such applications, the flow is typically generated by a localized heating of the interface or inhomogeneous spreading of a surfactant, and is regulated by the energy input or the diffusion rate of the surfactant. Concentration gradients at interfaces show particular potential for the emergence of complex behavior in solution. Earlier, our group has shown that self-organizing droplet-filament networks can be obtained between a surfactant source and attractive drain droplets, when the Marangoni flows between them are carefully mediated ${ }^{28}$. Furthermore, it has been demonstrated that surface tension gradients can be used to identify the shortest path through a maze ${ }^{29,30}$, offering the possibility to guide passive objects through a complex topology. Light has also been investigated as an external stimulus to remotely control these interfacial flows. While it has been used directly to locally heat an interface ${ }^{31}$, recent works have shown its potential when combined with photo-switchable surfactants ${ }^{32,33}$ for the manipulation of $\mu \mathrm{m}$ range objects. In that case, light intensity and surfactant concentration were used to regulate the flow velocity ${ }^{34-36}$.

The potential of Marangoni flows to directionally transfer matter prompted us to explore how these flows can be introduced in and coupled to out-of-equilibrium chemical reaction systems. In this work, we unravel how the coupling of this surface phenomenon with a chemical reaction can generate self-sustained flows, that are driven by a chemical reaction and - in turn - deliver the reagents for this reaction. In our system, a surface tension gradient is established by a photo-controlled chemical reaction that consumes surfactant at the interface, and thereby maintains the system out-of-equilibrium (Figure 1). We introduce in solution a photoacid generator, 1-(2-nitrophenyl)ethyl sulphate (NPE) and a $\mathrm{pH}$-responsive surfactant, sodium oleate (Fig. $1 \mathrm{~b})$ that react when the system is locally irradiated. Indeed, the photodissociation of NPE, triggered by UV exposure, causes an acidification of the solution, which in turn results in the conversion of sodium oleate into oleic acid (OA) when the local $\mathrm{pH}$ drops below the pKa of the surfactant (Fig. 1c). The accumulation of water-immiscible oleic acid into phase-separated droplets in the UV exposed area depletes the interface and the aqueous bulk of surfactant, and thereby establishes a surface tension gradient. In these conditions, a Marangoni flow emerges in the system (Fig. 1a,d), mass is transferred from the high $\mathrm{pH} /$ low surface tension non-irradiated area towards the low $\mathrm{pH} /$ high surface tension area, bringing unreacted NPE and oleate towards the UV source. We demonstrate that chemical reactions are the main driver of the Marangoni flow and that directional, long-lived interfacial flows 
a
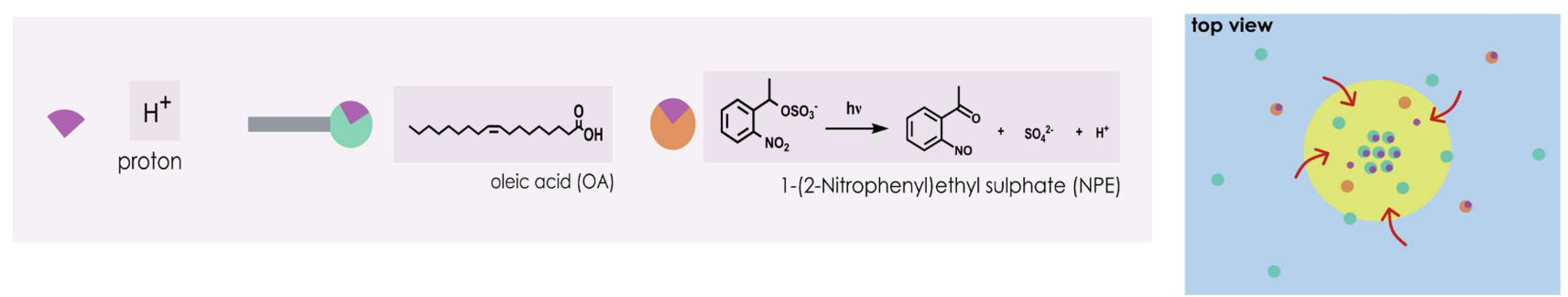

b

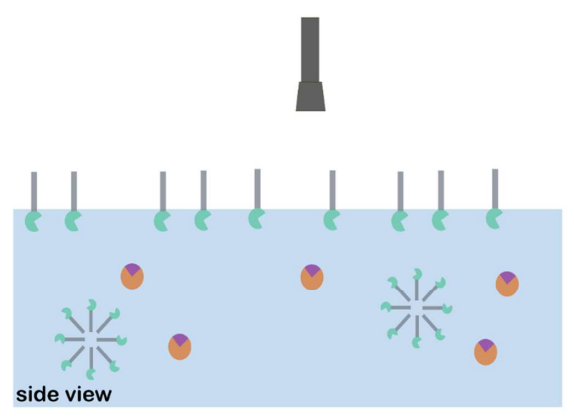

C

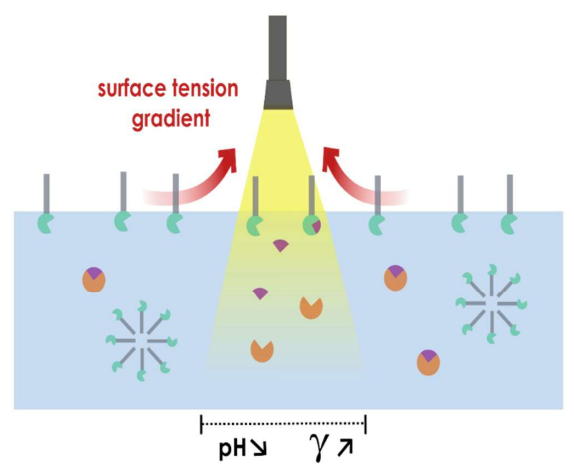

d

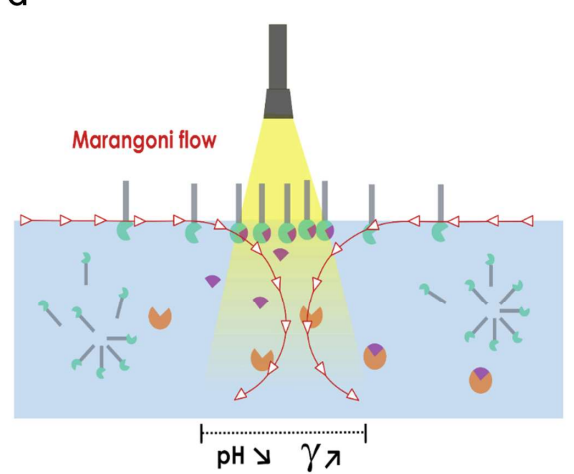

Figure 1. Self-sustained Marangoni flow triggered by localized UV exposure. (a) and (b) An interfacial flow can be generated in solutions containing the photoacid generator 1-(2-nitrophenyl)ethyl sulphate (NPE) and the surfactant sodium oleate. (c) Local UV irradiation results in the release of protons in solution and therefore the conversion of oleate into oleic acid, resulting in an increase in surface tension $\gamma$. (d) The consumption of the surface active sodium oleate leads to a surface tension gradient, which eventually generates a Marangoni flow towards the area exposed to UV.

can be obtained by mediating this reaction, which relies on the delivery of reagents by the flow. As long as the system is kept outof-equilibrium by the combination of UV irradiation and supply of new surfactant and photoacid, the Marangoni flow is sustained.

\section{Results and Discussion}

\subsection{The experimental system.}

To establish a self-sustained Marangoni flow, we locally expose (approx. $3 \mathrm{~mm}^{2}$ area) aqueous solutions of 1-(2-nitrophenyl)ethyl sulphate (NPE) and sodium oleate to UV $(\lambda=365 \mathrm{~nm})$, subsequently inducing the irreversible dissociation of NPE into 2'nitroacetophenone, a sulphate ion and a $\operatorname{proton}^{37,38}$ (Fig. 1). As optical microscopy images reveal (Fig. 2a-f and Video S1), microdroplets of OA appear at the air-water interface within seconds after the onset of UV exposure (Fig. 2b). These droplets form a cluster within the irradiated zone, which rapidly grows during the first 130 seconds (Fig. 2c). Right after that, the cluster surprisingly shrinks by a factor six and appears darker (Fig. 2d). Microscope observations at higher magnification (Fig. $2 \mathrm{~g}-\mathrm{h}$ and Video S2) show a fast merging of droplets, resulting in a denser and visually darker droplet cluster. The droplet cluster remains in this shrunken state for approximatively 200 seconds and eventually expands across the interface (Fig. 2e-f). The evolution of the cluster over time is tracked by image analysis, and systematically displays a typical pattern of subsequent growth, shrinkage and expansion phases (Fig. 2i). The transition from the growth to the shrinkage phase can be explained by the destabilization of the oleic acid in oleate emulsion. Indeed, the droplets of oleic acid are momentarily stabilized in a surfactant rich medium, but as oleate is locally depleted, the emulsion is destabilized and the droplets eventually merge. This effect is also enhanced by the flow that brings the droplets closer together, increasing the probability of merging events.

The different phases prompted us to assess the air-water surface tension kinetics in parallel with the time-dependent observations in optical microscopy. Using a Wilhelmy plate setup, the evolution of the surface tension is measured over time (Fig. 2j). A linear rise is observed during the first 130 seconds, matching with the growth phase of the cluster and indicative of a depletion of the oleate surfactant from the air-water interface. During the subsequent shrinkage phase, the surface tension steadily increases. After approx. $300 \mathrm{~s}$ - coinciding with the onset of the cluster expansion phase - the increase in surface tension levels off at $\Delta \gamma=+5.2 \mathrm{mN} \mathrm{m}^{-1}$, indicating that the depletion of oleate from the air-water interface is completed and hence no further flow towards the UV exposed area occurs.

Notably, UV irradiation plays a crucial role in driving the surface tension gradient in the system. The rapid release of protons at the irradiation area, as was observed by the color change of $\mathrm{pH}$ indicator Congo red (Video S3), ensures that the consumption of oleate is continuous and faster than the diffusion of oleate from the bulk to the interface. Indeed, in experiments where UV is sequentially turned on and off every 30 seconds, we observe the OA cluster increasing in size when exposed to UV, and when UV is turned off instantly shrinking and drifting away from the irradiated area (Fig. S2 and Video S4). This observation indicates that the flow is driven by the reaction between the surfactant and the protons generated by the photoacid, and that this reaction is sustained by the flow of reagents towards the irradiation area. 
a

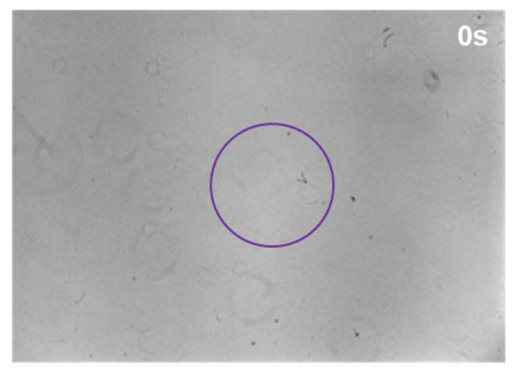

d

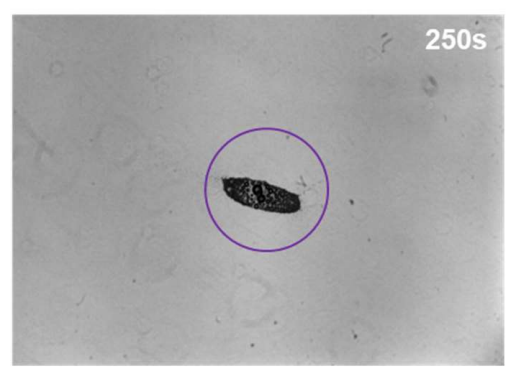

b

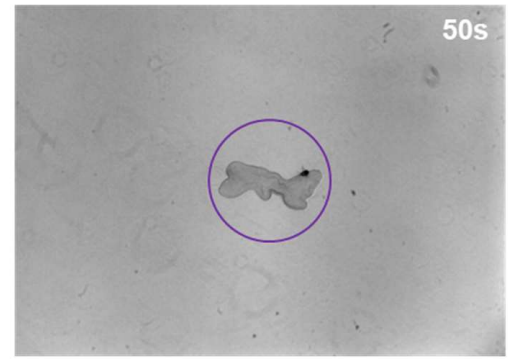

e

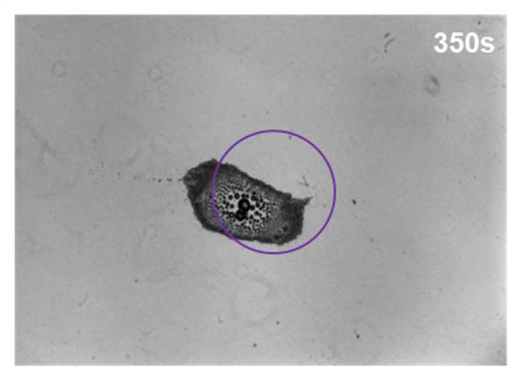

c

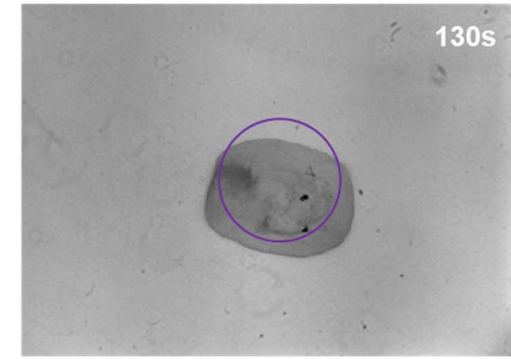

$\mathbf{f}$

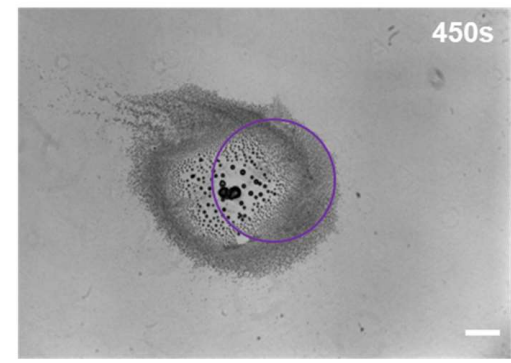

$\mathbf{g}$

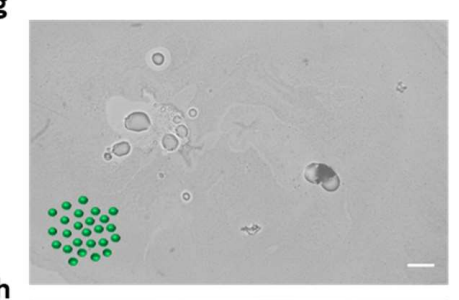

h

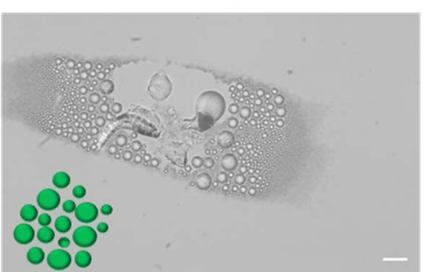

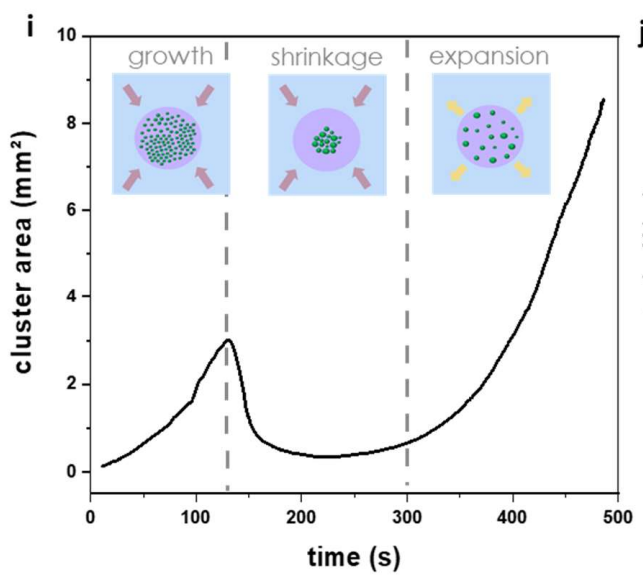

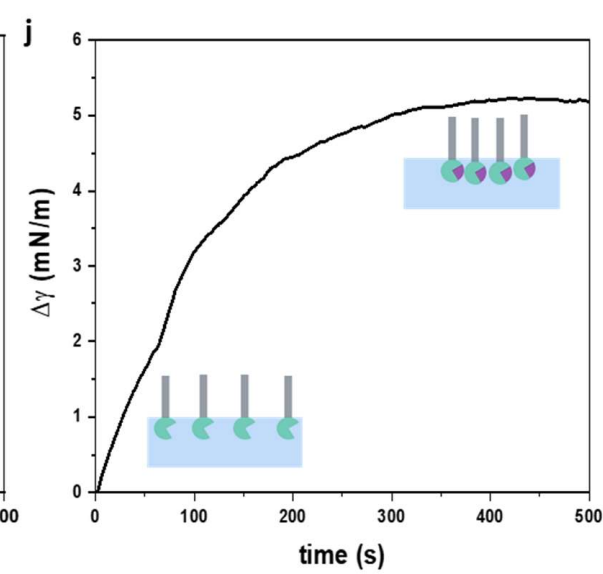

Figure 2. Generation of a self-sustained Marangoni flow in a sodium oleate/NPE solution. (a-f) Optical microscopy recording of an oleate/NPE solution that is locally exposed to UV (the purple circle). A cluster of oleic acid droplets appears at the air-water interface within a few seconds after the onset of UV exposure (at $t=0 \mathrm{~s}$ ), and grows until the surface tension reaches a new equilibrium. [NPE $]_{0}=1.4 \mathrm{mM}$; [oleate $]_{0}=0.078 \mathrm{mM}$, scale bar $=0.5 \mathrm{~mm}$. $(\mathrm{g})$ and $(\mathrm{h})$ Optical microscopy recording of an oleate/NPE solution at $10 x$ magnification, at $t=150 \mathrm{~s}(\mathrm{~g})$ and $t=230 \mathrm{~s}(\mathrm{~h})$, scale bar $=0.1 \mathrm{~mm}$. (i) Area of oleic acid droplet cluster vs. time for the experiment shown in a-f. (j) Surface tension $(\Delta \gamma)$ vs. time, measured with a Wilhelmy plate setup upon exposing the NPE/sodium oleate solution to UV from $t=0 \mathrm{~s}$ onwards.

\subsection{Simulations based on a kinetic model.}

To unravel how the flow depends on photo-generation of acid, as well as the protonation of the oleate surfactant, we have developed a simple kinetic model. As schematically represented in Figure $3 a$, the system is divided into a section 1 that is not exposed to UV and a section 2, exposed to UV. The irreversible photodissociation of the photoacid (PA) into a strong acid $(A)$ is set to occur exclusively in section 2 (reaction 1 ). The reversible diffusion of oleate from the aqueous bulk $\left(\mathrm{O}_{\mathrm{w}}\right)$ towards the airwater interface $\left(\mathrm{O}_{\text {int }}\right)$ is set throughout the whole system and we define a finite number of sites $\theta$ at the interface that the surfactant can occupy (reaction 2). The formation of oleic acid (OA) from the protonation of $\mathrm{O}_{\mathrm{w}}$ and $\mathrm{O}_{\text {int }}$ only occurs when the $\mathrm{pH}$ is below the $\mathrm{pKa}$ of OA. This means that the rate constants of reaction 3 and 4 are established to reflect the $\mathrm{pH}$-dependence of these reactions (see Supplementary Information). Because oleic acid is immiscible with water, $\mathrm{OA}$ is considered to be present in the system in the form of phase-separated droplets at the interface. Therefore, the deprotonation of OA is not taken into consideration in this system. Finally, the flow factor is defined as $\varphi$ and assumed to be proportional to the difference in the concentration of oleate at the interface $\Gamma_{\text {Oint }}$ between section 1 and 2 (equation 5). As elaborated on in the Supplementary Information, the flow transfers the content of section 1 to section 2 - including the content of the air-water interface. In turn, the back flow transfers an equal volume of the bulk fluid back from section 2 to section 1 . We used the kinetic model to simulate irradiation of section 2 at $t=0 \mathrm{~s}$ and from there, observe the evolution of the concentration of the different compounds in section 1 (blue lines) and 2 (orange lines) over a time course of $600 \mathrm{~s}$ (Fig. 3b-f). The concentration in PA drops within seconds in section 2, and the generated acid depletes $\mathrm{O}_{\mathrm{w}}$ and $\mathrm{O}_{\text {int }}$ upon protonation. The concentrations in PA, 

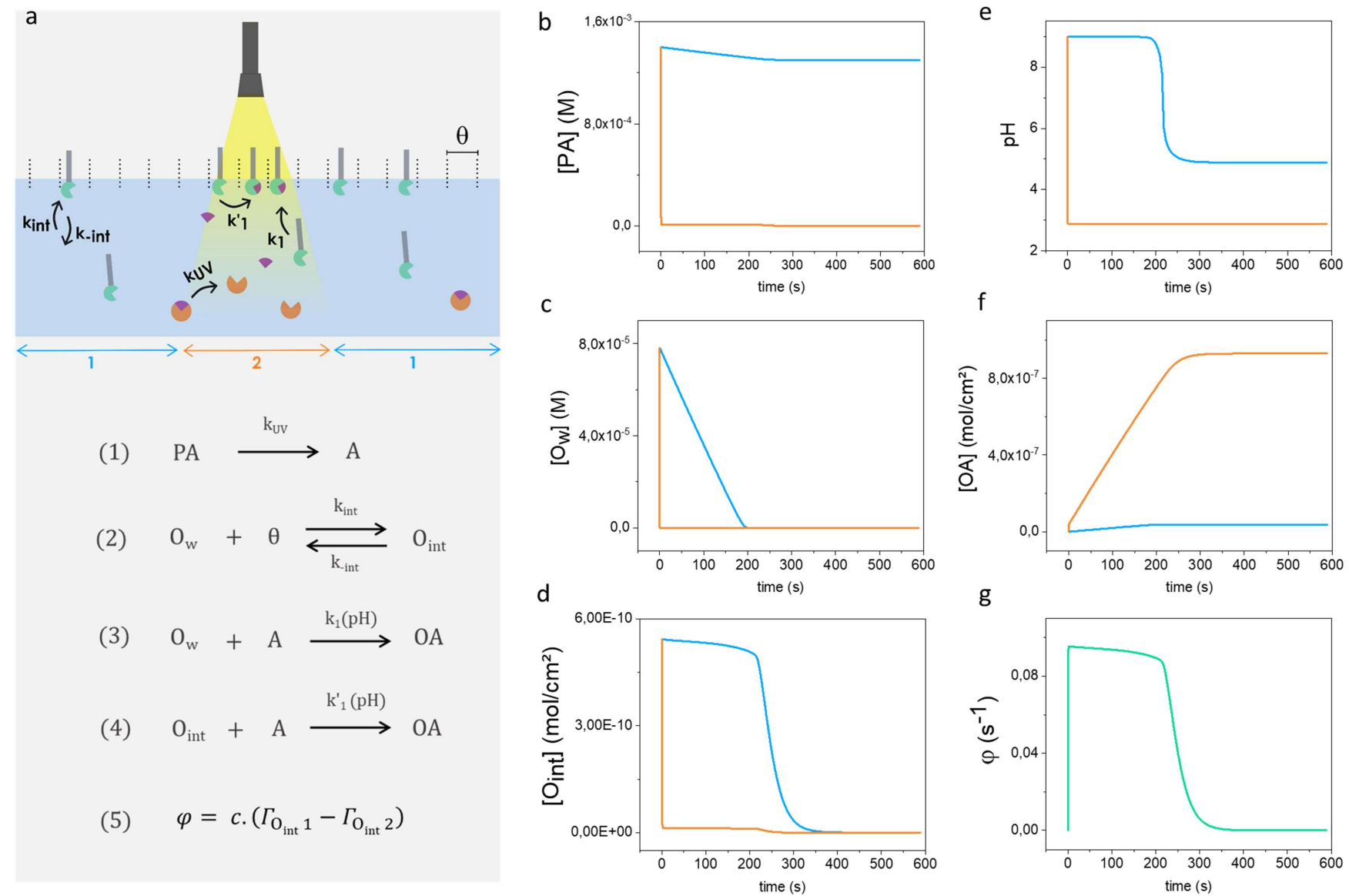

Figure 3. Simulation of the emergence of a self-sustained Marangoni flow with a kinetic model. (a) Schematic representation of the kinetic model describing the diffusion of oleate from the bulk $\left(\mathrm{O}_{\mathrm{w}}\right)$ to the interface $\left(\mathrm{O}_{\text {int }}\right)$, the dissociation of the photoacid $(\mathrm{PA})$ under UV irradiation and the formation of acid $(\mathrm{A})$ and oleic acid (OA). The simulation runs from $t=0 \mathrm{~s}$ (UV on) to $t=600 \mathrm{~s}$, the concentrations of $\mathrm{PA}(\mathrm{b}), \mathrm{O}_{\mathrm{w}}(\mathrm{c}), \mathrm{O}_{\text {int }}(\mathrm{d})$ and $\mathrm{OA}(\mathrm{f})$ are obtained along with pH (e) in sections 1 (blue curves) and 2 (orange curves). The flow factor, calculated from equation 5 , is displayed in (g).

$\mathrm{O}_{\text {int }}$ and $\mathrm{O}_{\mathrm{w}}$ remain very low over time in this section, indicating that these reagents are immediately consumed. The large concentration difference between $\mathrm{O}_{\text {int }}$ in sections 1 and 2 provides the driving force for the emerging flow (Fig. $3 \mathrm{~g}$ ), which is steadily sustained as long as oleate is available to be transferred to the UV exposed section. We also observe the OA concentration to rise in section 2 from $t=0 \mathrm{~s}$ and reach a plateau after all oleate is depleted from the system.

Furthermore, our simulations reveal intriguing effects of the oleate and photoacid concentration on the emergence of the selfsustained Marangoni flow. Figure $4 a$ plots $\varphi_{\max }$, defined as the maximum flow factor, against [oleate $]_{0}$ and $[\mathrm{PA}]_{0}$. In conditions where [oleate $]_{0}>[\mathrm{PA}]_{0}$, the model predicts the flow to be negligible. As shown in Figure 4c, a threshold is passed when [oleate $]_{0} \approx[\mathrm{PA}]_{0}-$ i.e. $R_{0} \approx 1$, where $R_{0}$ is defined as the ratio between the initial sodium oleate and photoacid concentration (i.e. $R_{0}=$ [oleate $\left._{0} /[\mathrm{NPE}]_{0}\right)$. Below the threshold, $\varphi_{\max }$ increases when $[P A]_{0}$ is increased for a fixed [oleate] $]_{0}$. In opposition, $\varphi_{\max }$ decreases when [oleate $]_{0}$ is increased for a fixed $[\mathrm{PA}]_{0}$. The transition between flow and no flow around $R_{0}=1$ can be rationalized by considering how the [oleate $]_{0} /[\mathrm{PA}]_{0}$ ratio affects the oleate concentration at the interface in section $1\left(\mathrm{O}_{\text {int1 }}\right)$ and section $2\left(\mathrm{O}_{\text {int2 }}\right)$. In both areas, $\mathrm{O}_{\text {int }}$ is regulated by the equilibrium between the aqueous bulk and the interface (eq. 2, Fig. 3a). The acid that is generated in section 2 depletes the oleate upon protonation, however, in conditions where [oleate $]_{0}>[\mathrm{PA}]_{0}$, the oleate concentration at the interface remains homogeneous despite exposure to UV ( $\Gamma_{\text {Oint } 1} \approx \Gamma_{\text {oint } 2}$, Fig. S3 $)$. Under these conditions, the diffusion of $\mathrm{O}_{\mathrm{w}}$ to the interface compensates for the local depletion of $\mathrm{O}_{\text {int }}$, thereby preventing the establishment of a surface tension gradient and the emergence of a Marangoni flow. When $[\mathrm{PA}]_{0}>[\text { oleate }]_{0}$, all oleate in section 2 can be depleted, such that $\Gamma_{\text {oint } 1}>\Gamma_{\text {oint } 2}$, providing the driving force for the flow (Fig. S3). Under these conditions, the flow velocity is dependent on the difference between these two concentrations, and a maximum $\varphi$ is reached when $\Gamma_{\text {Oint }} 2$ is at a minimum (Fig. $3 d)$. Increasing $[P A]_{0}$ promotes the depletion of $\mathrm{O}_{\mathrm{int} 2}$, eventually leading to higher velocities. In contrast, the flow factor is reduced when increasing [oleate] 0 , because it stabilizes $\mathrm{O}_{\text {int }}$.

The flow duration, calculated from $t=0 \mathrm{~s}$ to the time at which $\varphi$ $=0.01 \cdot \varphi_{\max }$, is plotted against [oleate $]_{0}$ and $[\mathrm{PA}]_{0}$ in Figure $4 \mathrm{~b}$. Increasing the amount of oleate increases the time for which the flow is sustained - under the condition [oleate $]_{0}<[P A]_{0}$. This can be rationalized by the fact that the flow can be sustained as long as there is oleate available in the non-irradiated section, to maintain the surface tension gradient. The presence of photoacid has the opposite effect on the flow duration: increasing $[P A]_{0}$ implies a faster depletion of the oleate surfactant reserve. The interdependence between flow duration, velocity and $R_{0}=$ [oleate $]_{0} /[\mathrm{PA}]_{0}$ is presented in Figure 4c, which features a 'flow' 


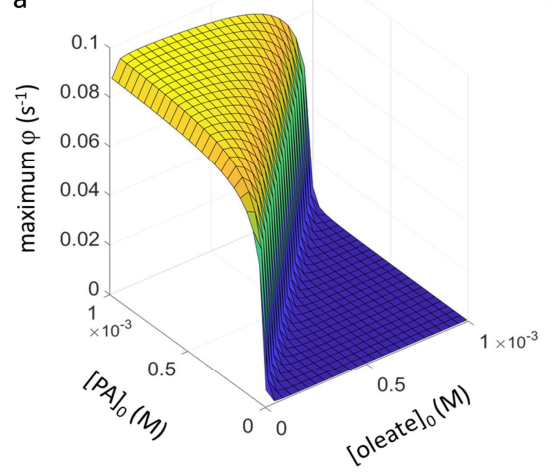

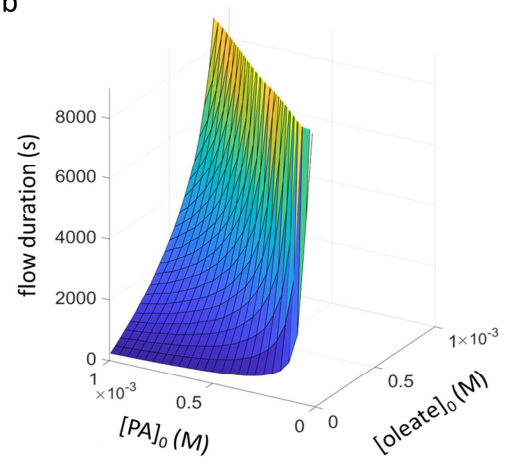

C

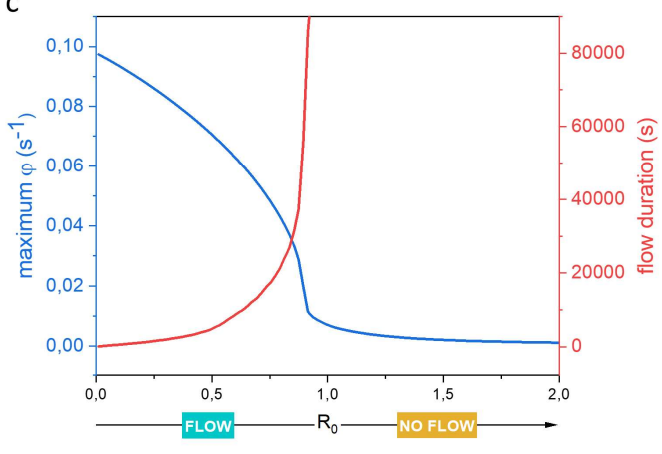

Figure 4. Simulations of maximum flow factor $\varphi(\mathrm{a})$ and flow duration (b) depending on initial reagent concentrations ([PA $]_{0}$ and [oleate $\left.]_{0}\right)$. (c) These same flow parameters are plotted against $R_{0}$, where [oleate $]_{0}=0.078 \mathrm{mM}$, and $[\mathrm{PA}]_{0}$ is varied.

regime at $R_{0}<1$, and a 'no flow' regime when $R_{0}$ increases above 1. Together, these results imply that the surfactant acts as the fuel for the self-sustained reaction, which attracts new reagents as long as fuel is available. The photoacid acts as a regulator and the excess of photoacid over surfactant determines the rate at which the fuel is dissipated.

\subsection{Establishing the Marangoni flow beyond a threshold in ratio of reagent concentrations.}

Guided by the results obtained from the simulations with the model, we have experimentally studied the emergence of the Marangoni flow for different concentration ratios of NPE and sodium oleate. The flow velocity, which is proportional to the flow factor $\varphi$, was measured as a function of $R_{0}$. Hydrophobic poly(methyl methacrylate) particles floating at the air-water interface were used as tracers to measure the velocity. The influence of the initial photoacid concentration was first assessed by performing a series of experiments at constant sodium oleate concentration and increasing NPE concentration, ranging from $R_{0}$ $=5$ to 0.01 (Fig. 5). Upon UV irradiation at high $R_{0}$ values, the tracer particles drift across the interface at low velocity and without particular trajectory (Fig. $5 \mathrm{~b}$ and Video S5). At low $R_{0}$, however, the particles migrate towards the UV exposed area at a higher velocity (Fig. 5c and Video S6). These observations are confirmed by a systematic analysis of the average particle velocity vs. $R_{0}$ (Fig. 5a). The transition from random particle motion to directional flow occurs when $R_{0}$ approaches 1 ; below this value, the particle velocity increases sharply with the initial NPE concentration, with particles moving at a rate of $0.05 \mathrm{~mm} \mathrm{~s}^{-1}$ for $R_{0}=0.5$ up to $0.44 \mathrm{~mm} \mathrm{~s}^{-1}$ for $R_{0}=0.01$.

To explore further the effect of photoacid concentration on the flow velocity, we compare the evolution of surface tension in the system at different $R_{0}$ values (Fig. $5 \mathrm{~d}-\mathrm{e}$ ). At $R_{0}=0.01$, UV irradiation triggers an instant rise in surface tension, demonstrating that a surface tension gradient can be efficiently established in such conditions. The first $50 \mathrm{~s}$ of the UV exposure gives a particularly sharp linear rise in surface tension, with a 0.11 $\mathrm{mN} \mathrm{m}{ }^{-1} \mathrm{~s}^{-1}$ slope. We observed that the surface tension kinetics show a diminishing slope with increasing $R_{0}$ (Fig. S4), and with $R_{0}$ $=1$, the surface tension remains unaffected upon irradiation (Fig. $5 \mathrm{~d})$. The correlation between the measured flow velocity and a change in surface tension agrees with earlier reported relationships between velocity and surface tension gradient ${ }^{39}$, and corroborates that the emergence of flow is driven by the depletion of oleate from the interface.

The impact of the initial oleate concentration on flow velocity was also studied in a series of experiments at constant NPE concentration and different sodium oleate concentrations (Fig. S5). Increasing the oleate concentration leads to a decrease in flow velocity; a larger concentration of oleate in the aqueous bulk implies that despite a rapid depletion, the interface remains rich in oleate due to diffusion of surfactant molecules from the bulk, thus countering the establishment of surface tension gradients significant enough to drive the flow.

\subsection{The antagonistic effect of photoacid and surfactant reagents on the Marangoni flow.}

The duration for which the flow can be sustained is impacted by the chemistry of the system. We have investigated the influence of [NPE $]_{0}$ and [oleate] $]_{0}$ on flow duration, which is estimated based on image analyses of the experimentally observed OA droplet cluster time profiles. Here, we consider that the flow is no longer sustained as soon as the cluster starts expanding, or leaves the UV exposed area (see Experimental section and Figs. S6-7 for details). Figure $6 a, c$ highlights the influence of [NPE] on the flow duration, at a constant oleate concentration. The flow duration decreases with the initial photoacid concentration, lasting more than 20 minutes at concentrations below $0.5 \mathrm{mM}$, to only 3 minutes when increased to $2.8 \mathrm{mM}$. The influence of [oleate] $]_{0}$ on flow duration, while keeping [NPE] $]_{0}$ constant, is opposite to the effect of $[\mathrm{NPE}]_{0}$, as shown in Figure $6 \mathrm{~b}, \mathrm{~d}$. The flow duration increases with oleate concentration, from 2 minutes when [oleate] $_{0}=0.016 \mathrm{mM}$ to 25 minutes when raised to $0.65 \mathrm{mM}$. Further increasing the oleate concentration does not extend the time for which the flow is sustained, as under these conditions $R_{0}$ $>0.5$ the Marangoni flow becomes very weak and unstable (as shown in Fig. 5a), leading to a high variation in flow duration. This instability in the system is exemplified by the behavior of the OA droplet cluster, drifting away from the UV exposed area instead of showing the expected growth-shrinkage-expansion pattern (Video S7). 
Taken together, these observations can be correlated back to $R_{0}$ $=[\text { oleate }]_{0} /[\mathrm{NPE}]_{0}$, as shown in Figure 6e. Even though both NPE and oleate are essential to establish the flow, these reagents have antagonistic effects on the duration and intensity of the flow - as predicted by the simulations with the model. The flow duration increases with $R_{0}$, however, the flow velocity decreases with $R_{0}$ (Fig. 5a): larger concentrations of NPE (small $R_{0}$ ) result in higher flow velocities, whereas the flow is sustained for a shorter period of time and, vice versa, larger concentrations of oleate result in lower velocities and longer flow durations. a

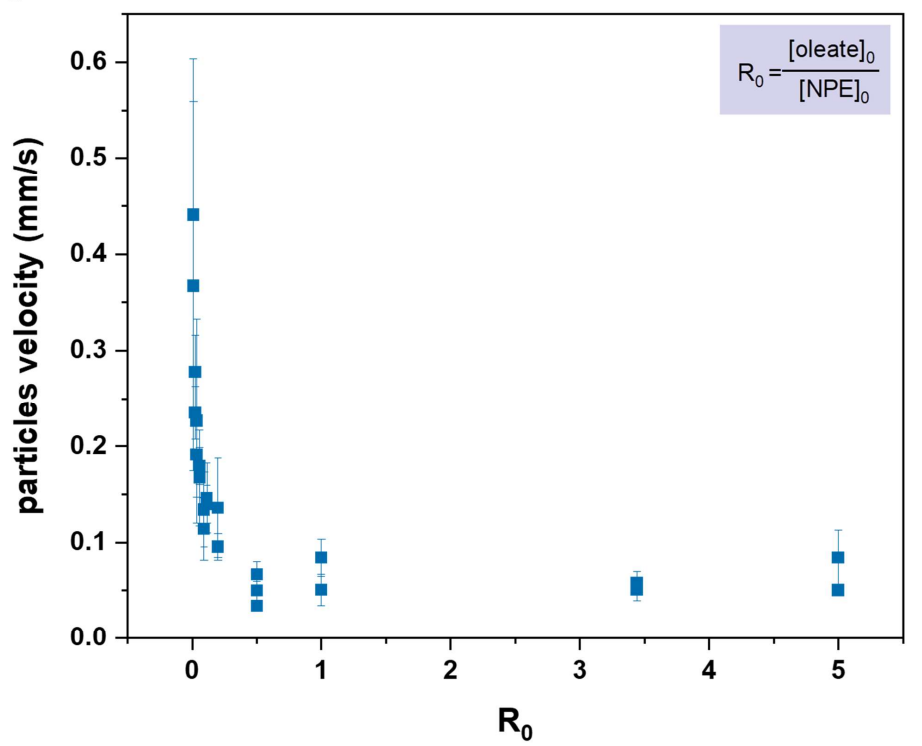

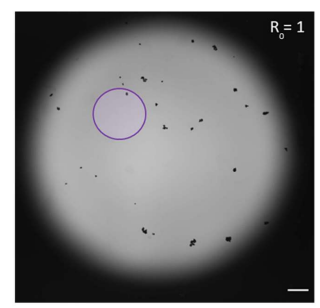

c

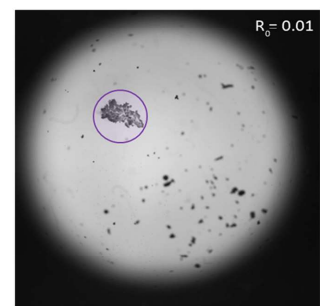

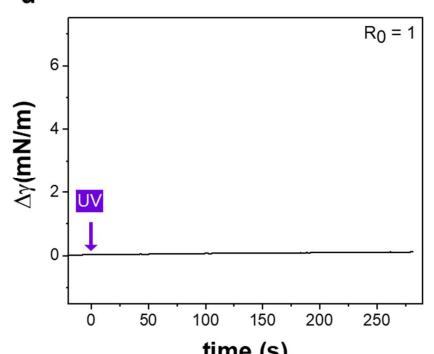

e

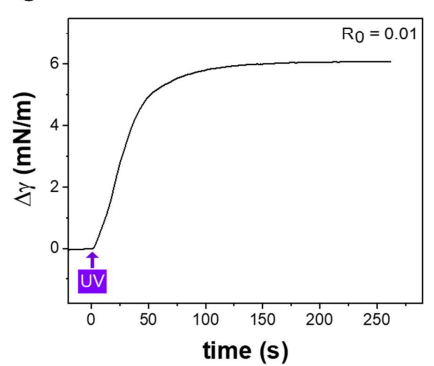

Figure 5. Influence of initial concentrations of the reagents on flow velocity. $R_{0}$ is defined as the ratio [oleate] $/[\mathrm{NPE}]_{0}$, for all samples [oleate] is fixed at $0.078 \mathrm{mM}$ while $[N P E]_{0}$ is varied. (a) Hydrophobic PMMA particles are applied at the air-water interface to track the flow velocity. The average velocity of the particles is measured for oleate/NPE solutions under UV irradiation. (b) and (c) Microscopy images of oleate/NPE solutions at different $R_{0}$ values. Clustering of the tracer particles in the irradiation area is observed for $R_{0}=0.01$, while they move randomly at the interface for $R_{0}=1$. The purple circle represents the UV exposed area, the scale bar represents $1 \mathrm{~mm}$. (d) and (e) Surface tension $(\Delta \gamma) v s$. time, measured for solutions at respectively $R_{0}=1$ and $R_{0}=0.01$, upon continuous UV exposure from $t=0 \mathrm{~s}$.

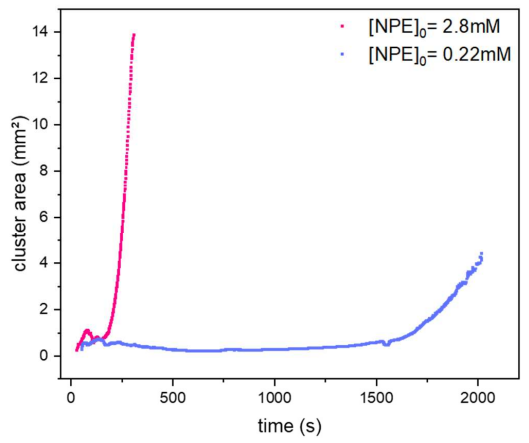

C

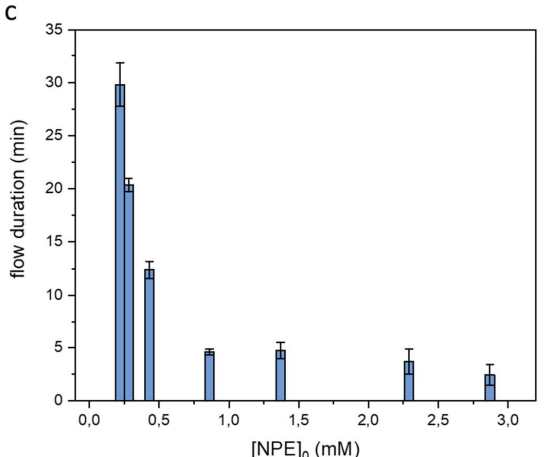

b

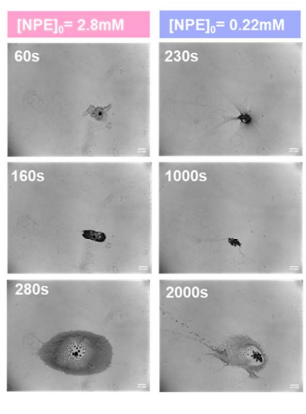

d

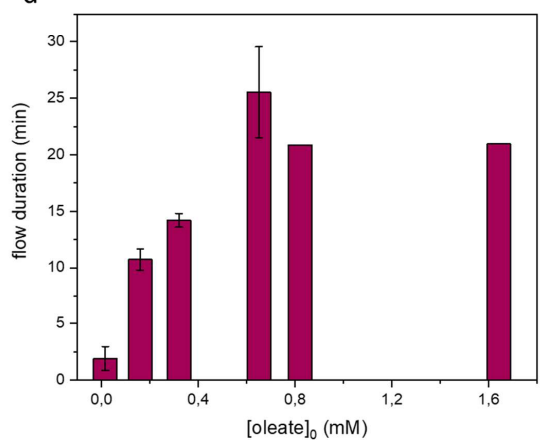

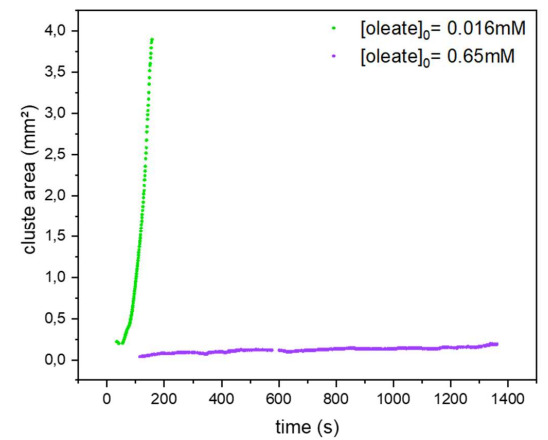
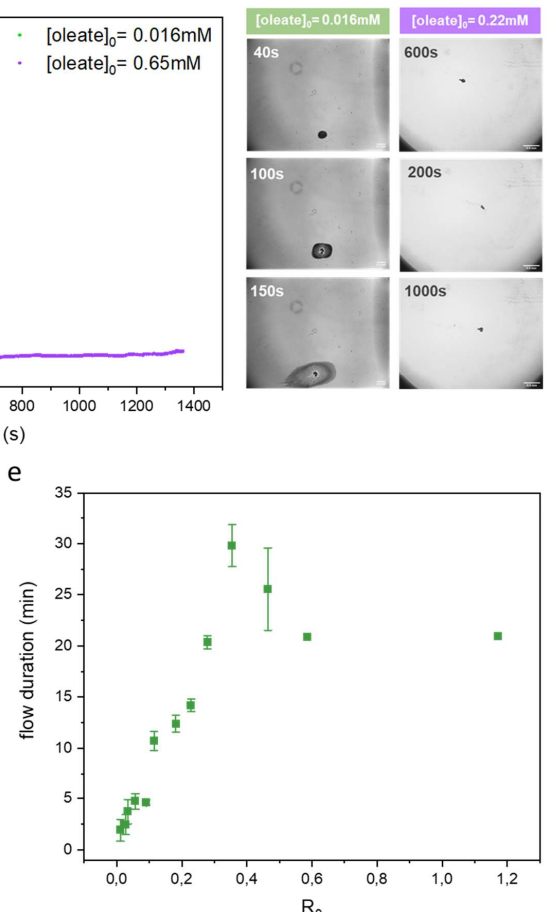

Figure 6. Influence of reagent concentrations on flow duration. (a) OA droplet cluster area vs. time and corresponding optical microscopy recording (scale bar $=0.5$ $\mathrm{mm}$ ) at $[\mathrm{NPE}]_{0}=0.22 \mathrm{mM}$ and $2.8 \mathrm{mM}$ (with [oleate $]_{0}=0.078 \mathrm{mM}$ ). (b) OA droplet cluster area $v$ s. time and corresponding optical microscopy recording (scale bar $=0.5 \mathrm{~mm}$ ) at [oleate $]_{0}=0.016 \mathrm{mM}$ and $0.22 \mathrm{mM}$ (with $[\mathrm{NPE}]_{0}=1.4 \mathrm{mM}$ ). (c) Flow duration against $[\mathrm{NPE}]_{0}$, with fixed [oleate] $]_{0}=0.078 \mathrm{mM}$ and $(\mathrm{d})$ flow duration against [oleate $]_{0}$, with fixed $[\mathrm{NPE}]_{0}=1.4 \mathrm{mM}$. (e) Flow duration vs. $R_{0}=$ [oleate $]_{0} /[\mathrm{NPE}]_{0}$. 

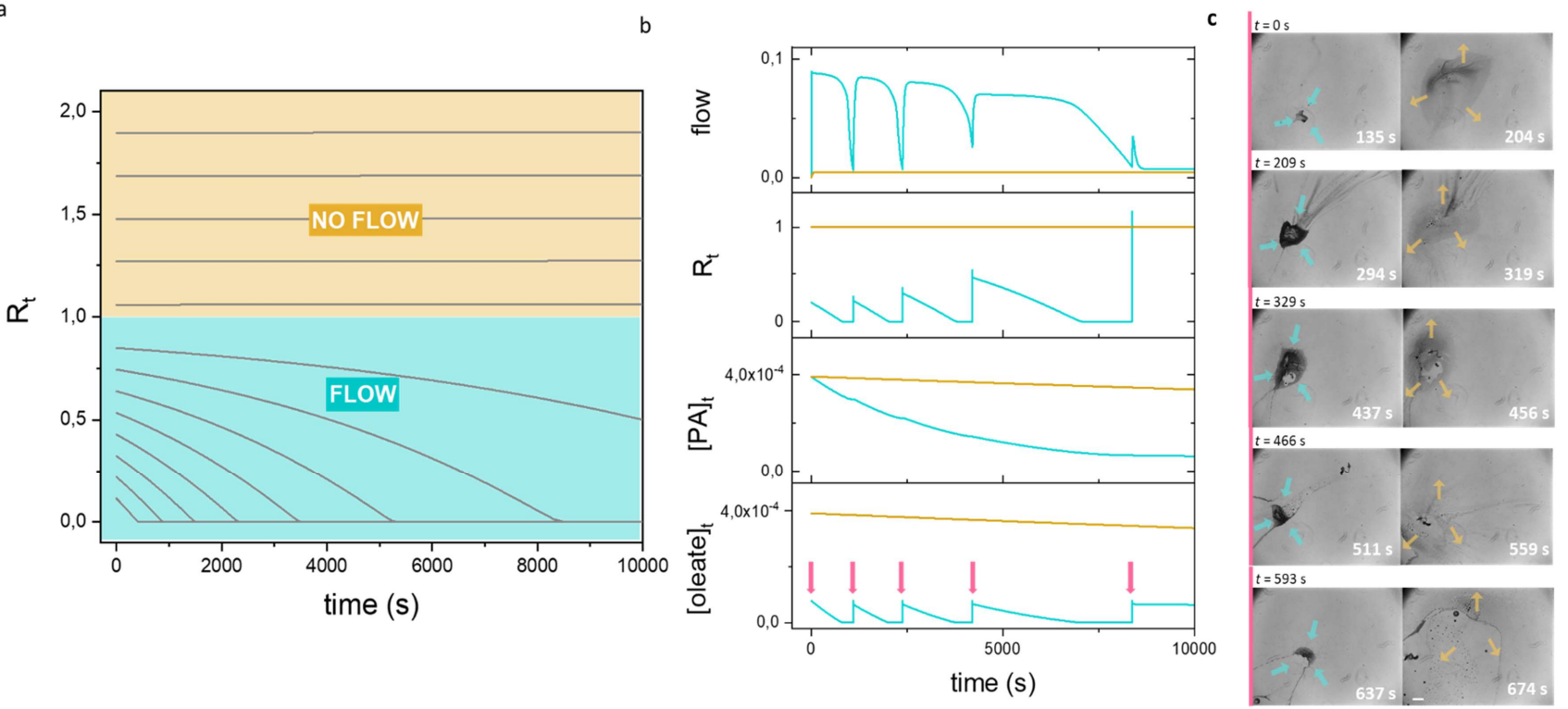

Figure 7. Simulation results displaying the influence of $R_{\mathrm{t}}$, defined as [oleate] $/[\mathrm{PA}]_{\mathrm{t}}$ on the system. (a) Evolution of $R_{\mathrm{t}}$ over time. (b) Impact of incremental addition of oleate on flow, $R_{\mathrm{t}}$, [PA] and [oleate]; the pink arrows represent the times at which the oleate fractions are added $(t=0 \mathrm{~s} ; 1096 \mathrm{~s} ; 2384 \mathrm{~s} ; 4204 \mathrm{~s}, 8373 \mathrm{~s} ;$ orange curves). The blue curves represent a simulation where all oleate is added at $t=0 \mathrm{~s}$. (c) Optical microscopy images of a oleate/NPE solution exposed to UV, where a sodium oleate solution $(19 \mathrm{mM})$ is added sequentially in $5 \mu \mathrm{L}$ fractions at $t=0 \mathrm{~s} ; 209 \mathrm{~s} ; 329 \mathrm{~s} ; 466 \mathrm{~s}$ and $593 \mathrm{~s}$, [NPE] $0=2.8 \mathrm{mM}(\mathrm{scale}$ bar $=0.5 \mathrm{~mm})$.

\subsection{Establishing self-sustained flow conditions.}

Finally, we revisited the conditions where a self-sustained Marangoni flow can be established, exploiting the threshold conditions required for the flow to start $\left(R_{0}<1\right)$. Figure 7 a shows the evolution of [oleate $]_{\mathrm{t}} /[\mathrm{PA}]_{\mathrm{t}}$, represented as $R_{\mathrm{t}}$, over time, as predicted by the model under continuous UV exposure. The initial ratio at which the reagents are introduced in the system determines whether it is possible to establish the flow: Indeed, no flow is predicted under conditions of $R_{\mathrm{t}}>1$ at $t=0 \mathrm{~s}$. In contrast, the simulations started in flow conditions (i.e. $R_{\mathrm{t}}<1$ ) display a steady decrease of $R_{\mathrm{t}}$ over time, showing that the flow is not only triggered by the initial surface tension gradient but sustains itself by bringing PA and oleate at the UV exposed area. In this regime, the rate at which $R_{\mathrm{t}}$ decays is directly related to the reserve of oleate in the system, suggesting that the flow duration can be extended by sequential addition of oleate. In Figure $7 \mathrm{~b}, \varphi$ vs. time is simulated in a scenario where sodium oleate is sequentially added in 5 equal fractions to the system (pink arrows) and all NPE is present at $t=0 \mathrm{~s}$. After the first oleate addition at $t=0 \mathrm{~s}, R_{\mathrm{t}}$ starts at a value of 0.2 , and the flow is steadily sustained until it starts declining around $t=1000 \mathrm{~s}$. Subsequent additions of oleate bring the flow anew to its initial intensity, however, after every new addition, $R_{\mathrm{t}}$ is raised to a higher value. After the $5^{\text {th }}$ addition, $R_{\mathrm{t}} \approx$ 1 , and as a result the flow stops. Importantly, addition of all oleate at once at $t=0 \mathrm{~s}$, rather than in 5 fractions, impairs the flow to emerge (Fig. $7 b$ ). Similar results are obtained experimentally when re-supplying the medium with oleate in subsequential additions (Fig. 7c and Video S8). These results illustrate that even though oleate fuels the self-sustained flow, addition of oleate can only sustain the out-of-equilibrium system when supplied in the right parameter window (i.e. $R_{\mathrm{t}}<1$ ); when oleate is present in excess to the photoacid, the flow cannot start, as shown in Figure 5.

\section{Conclusion}

In summary, we have shown that a Marangoni flow can be generated from a chemical reaction between a photoacid generator and a $\mathrm{pH}$-responsive surfactant at the air-water interface when exposed to UV. We have demonstrated that such flow is first triggered by the surface tension gradient induced by the local depletion of sodium oleate, and then self-sustained as it continuously brings reagents to the UV exposed area. This outof-equilibrium process was observed and studied experimentally and rationalized by developing a simple kinetic model. Our analysis revealed the antagonistic effect of the reagents on flow parameters such as velocity and duration, with the photoacid generator acting as a system regulator and the oleate playing the role of a fuel for the system. We have also demonstrated that ensuring the presence of both reagents in the system is not sufficient to generate an interfacial flow and that the ratio $R_{0}$ in which they are introduced is crucial to guarantee a sustained surface tension gradient. Notably, we observed experimentally that while increasing the initial surfactant concentration led to flow sustained for more than 20 minutes, introducing an excess of oleate in the system, relative to the photoacid concentration, results in an absence of flow. This showed that in theory, as long as $R_{0}$ is kept below 1 , and both surfactant and photoacid are present, the Marangoni flow can be sustained for an indefinite duration at the interface. That possibility was explored by performing sequential additions of surfactant, and successfully showing that the flow duration could be extended.

These results open the possibility to use sodium oleate and other surfactants presenting similar $\mathrm{pH}$-responsiveness in combination with photoacid generators to design positive or negative triggers for the flow, and therefore to build flow-controlled feedback loops. Controlling the chemistry of such reaction-driven Marangoni flows becomes then an interesting approach to achieve complex behavior at air-water interfaces and within 
organic droplets ${ }^{40-42}$. Such insight is also useful to develop applications where Marangoni flows are used for propulsion ${ }^{43,44}$, self-assembly direction ${ }^{45-47}$ or to design systems with Marangonibased feedback mechanisms ${ }^{48}$.

\section{Acknowledgements}

We thank the Dutch Ministry of Education, Culture and Science (Gravitation program, 024.001.035) for financial support. We thank Arno van der Weijden, Jelle Prinsen, Mitch Winkens, Pieter de Visser and prof. Wilhelm Huck for stimulating discussions. We thank dr. Evan Spruijt for kindly providing the PHSA-g-PMMA particles used in the flow velocity analysis.

Keywords: out-of-equilibrium • Marangoni flow • fuel delivery • control mechanism

[1] J. C. Xavier, R. E. Gerhards, J. L. E. Wimmer, J. Brueckner, F. D. K. Tria, W. F. Martin, Commun. Biol. 2021, 4, 1-10.

[2] G. M. Whitesides, J. P. Mathias, C. T. Seto, Science 1991, 254, 13121319.

[3] A. N. Landge, B. M. Jordan, X. Diego, P. Müller, Dev. Biol. 2020, 460, 2-11.

[4] R. Merindol, A. Walther, Chem. Soc. Rev. 2017, 46, 5588-5619.

[5] T. Bánsági, A. F. Taylor, J. R. Soc. Interface 2018, 15, 20170945.

[6] S. N. Semenov, A. S. Y. Wong, R. M. van der Made, S. G. J. Postma, J. Groen, H. W. H. van Roekel, T. F. A. de Greef, W. T. S. Huck, Nat. Chem. 2015, 7, 160-165.

[7] S. N. Semenov, L. J. Kraft, A. Ainla, M. Zhao, M. Baghbanzadeh, V. E. Campbell, K. Kang, J. M. Fox, G. M. Whitesides, Nature 2016, 537, 656-660.

[8] A. A. Pogodaev, T. T. Lap, W. T. S. Huck, ChemSystemsChem 2021, 3, e2000033.

[9] B. Helwig, B. van Sluijs, A. A. Pogodaev, S. G. J. Postma, W. T. S. Huck, Angew. Chem. Int. Ed. 2018, 57, 14065-14069.

[10] T. Heuser, R. Merindol, S. Loescher, A. Klaus, A. Walther, Adv. Mater 2017, 29, 1606842.

[11] J. Boekhoven, W. E. Hendriksen, G. J. M. Koper, R. Eelkema, J. H. van Esch, Science 2015, 349, 1075-1079.

[12] H. Zhang, H. Zeng, A. Priimagi, O. Ikkala, Nat. Commun. 2019, 10, 3267.

[13] C. Perego, L. Pesce, R. Capelli, S. J. George, G. M. Pavan, ChemSystemsChem 2021, 3, e2000038.

[14] J. Leira-Iglesias, A. Tassoni, T. Adachi, M. Stich, T. M. Hermans, Nat Nanotechnol. 2018, 13, 1021-1027.

[15] M. Kumar, N. L. Ing, V. Narang, N. K. Wijerathne, A. I. Hochbaum, R. V. Ulijn, Nat. Chem. 2018, 10, 696-703.

[16] A. Mishra, S. Dhiman, S. J. George, Angew. Chem. Int. Ed. 2021, 60 , 2740-2756.

[17] S. Bal, C. Ghosh, T. Ghosh, R. K. Vijayaraghavan, D. Das, Angew. Chem. Int. Ed. 2020, 59, 13506-13510.

[18] M. A. Würbser, P. S. Schwarz, J. Heckel, A. M. Bergmann, A. Walther, J. Boekhoven, ChemSystemsChem 2021, 3, e2100015.

[19] V. K. Vanag, I. R. Epstein, Phys. Rev. Lett. 2001, 87, 228301.

[20] V. V. Yashin, A. C. Balazs, Science 2006, 314, 798-801.

[21] R. Yoshida, Adv. Mater. 2010, 22, 3463-3483.

[22] J. Horváth, I. Szalai, P. D. Kepper, Science 2009, 324, 772-775.

[23] G. D. Zissi, G. Angelis, G. Pampalakis, Chem SystemsChem 2021, 3, e2000018.

[24] R. Chen, S. Neri, L. J. Prins, Nat. Nanotechnol. 2020, 15, 868-874.

[25] Y. Zhang, S. Tsitkov, H. Hess, Nat. Catal. 2018, 1, 276-281.

[26] M. Roché, Z. Li, I. M. Griffiths, S. Le Roux, I. Cantat, A. Saint-Jalmes, H. A. Stone, Phys. Rev. Lett. 2014, 112, 208302.

[27] R. Sharma, T. E. Corcoran, S. Garoff, T. M. Przybycien, R. D. Tilton, Colloids Surf. Physicochem. Eng. Asp. 2017, 521, 49-60.
[28] A. van der Weijden, M. Winkens, S. M. C. Schoenmakers, W. T. S. Huck, P. A. Korevaar, Nat. Commun. 2020, 11, 4800.

[29] I. Lagzi, S. Soh, P. J. Wesson, K. P. Browne, B. A. Grzybowski, J. Am. Chem. Soc. 2010, 132, 1198-1199.

[30] K. Suzuno, D. Ueyama, M. Branicki, R. Tóth, A. Braun, I. Lagzi, Langmuir, 2014, 30, 9251-9255.

[31] M. Shono, S. Takatori, J. M. Carnerero, K. Yoshikawa, Appl. Phys. Lett 2020, 117, 073701.

[32] S. Chen, R. Costil, F. K.-C. Leung, B. L. Feringa, Angew. Chem. Int. Ed. 2021, 60, 2-26.

[33] P. Arya, D. Feldmann, A. Kopyshev, N. Lomadze, S. Santer, Soft Matter 2020, 117, 1148-1155.

[34] E. Chevallier, A. Mamane, H. A. Stone, C. Tribet, F. Lequeux, C. Monteux, Soft Matter 2011, 7, 7866-7874.

[35] S. N. Varanakkottu, S. D. George, T. Baier, S. Hardt, M. Ewald, M. Biesalski, Angew. Chem. 2013, 125, 7432-7436.

[36] C. Lv, S. N. Varanakkottu, T. Baier, S. Hardt, Nano Lett. 2018, 18, 6924-6930.

[37] A. Barth, J. E. T. Corrie, Biophys. J. 2002, 83, 2864-2871.

[38] S. Abbruzzetti, S. Sottini, C. Viappiani, J. E. T. Corrie, J. Am. Chem. Soc. 2005, 127, 9865-9874.

[39] T. Battal, C. D. Bain, M. Weiß, R. C. Darton, J. Colloid Interface Sci. 2003, 263, 250-260

[40] D. Babu, R. Scanes, R. Plamont, A. Ryabchun, F. Lancia, T. Kudernac S. P. Fletcher, N. Katsonis, 2020, ChemRxiv. Preprint. https://doi.org/10.26434/chemrxiv.12454100.v1

[41] Y. Xiao, S. Zarghami, K. Wagner, P. Wagner, K. C. Gordon, L. Florea, D. Diamond, D. L. Officer, Adv. Mater. 2018, 30, 1801821

[42] T. Banno, Y. Tanaka, K. Asakura, T. Toyota, Langmuir 2016, 32 9591-9597.

[43] B. Kwak, S. Choi, J. Bae, IEEE Robot. Autom. Lett. 2020, 5, 68296836.

[44] C. H. Meredith, P. G. Moerman, J. Groenewold, Y.-J. Chiu, W. K. Kegel, A. van Blaaderen, L. D. Zarzar, Nat. Chem. 2020, 12, 11361142

[45] X. Li, L. Chen, D. Feng, D. Weng, J. Wang, Cell Rep. Phys. Sci. 2020, $1,100220$.

[46] C. M. Jin, W. Lee, D. Kim, T. Kang, I. Choi, Small, 2018, 14, 1803055

[47] M. Cheng, G. Zhu, L. Li, S. Zhang, D. Zhang, A. J. C. Kuehne, F. Shi, Angew. Chem. Int. Ed. 2018, 57, 14106-14110.

[48] J. Grawitter, H. Stark, Soft Matter 2018, 14, 1856-1869. 